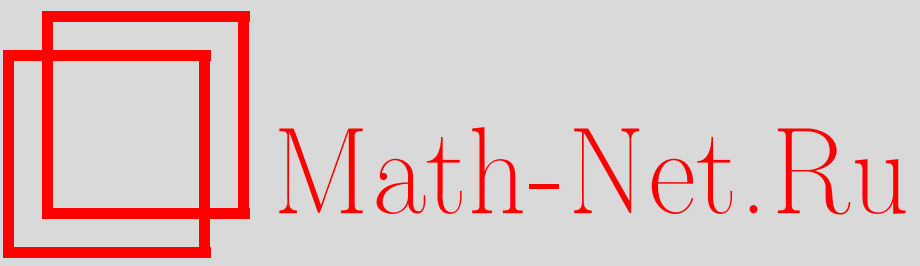

И. Н. Никитин, Квантовая теория струны в индефинитном пространстве состояний, ТМФ, 1996, том 107, номер 2, 213-228

DOI: https://doi.org/10.4213/tmf1150

Использование Общероссийского математического портала Math-Net.Ru подразумевает, что вы прочитали и согласны с пользовательским соглашением

http://www.mathnet.ru/rus/agreement

Параметры загрузки:

IP: 54.198 .64 .247

26 апреля 2023 г., 15:18:54 
ТЕОРЕТИЧЕСКАЯ

И МАТЕМАТИЧЕСКАЯ

ФИЗИКА

Том 107, № 2

май, 1996

И.Н. Никитин

\section{КВАНТОВАЯ ТЕОРИЯ СТРУНЫ В ИНДЕФИНИТНОМ ПРОСТРАНСТВЕ СОСТОЯНИЙ}

Рассмотрена квантовая теория струны в индефинитном (псевдогильбертовом) фоковском представлении. Показано, что при определенном выборе вакуумного состояния в 4-мерной теории открытой бозонной струны сокращаются все аномалии. При этом оказываются замкнутыми алгебра репараметризационной симметрии (в ковариантном подходе) и алгебра вращений (в калибровке светового конуса). Индефинитность пространства состояний вносит в теорию ряд нефизических черт. Обсуждаются модификации изложенного метода, необходимые для построения теории с более реалистическими свойствами.

\section{ВВЕДЕНИЕ}

Пространства состояний с индефинитным скалярным произведением используются в релятивистских квантовых теориях для обеспечения их явной лоренц-ковариантности. При этом, как правило, физический сектор теории не содержит состояний с отрицательной нормой, эти состояния выполняют в теории вспомогательную роль. Иногда индефинитные пространства состояний вводятся для других целей.

В 1943 г. Дирак [1] и Паули [2] предложили использовать индефинитные пространства состояний для устранения расходимостей в квантовых теориях поля. Эта возможность интенсивно исследовалась в 50-60-е годы [3]. Был сформулирован ряд моделей, непротиворечивое квантование которых возможно (только) в индефинитных пространствах состояний. Трудность вероятностной интерпретации, присущую этим моделям, в некоторых случаях удалось преодолеть с помощью определенных ограничений на вид взаимодействия и начальные состояния задачи рассеяния.

В данной работе индефинитные пространства состояний используются для устранения аномалий в теории релятивистской струны. В теории струны при использовании стандартной фоковской реализации оператор квадрата массы содержит расходимость в виде суммы энергий “нулевых колебаний”. Как и в теориях поля, эта расходимость устраняется при фиксации нормального упорядочения произведений операторов. При квантовании свободных полей эта процедура обычно приводит к непротиворечивой теории. В теории струны оператор квадрата массы входит в состав бесконечномерной алгебры внутренней симметрии. При введении нормального упорядочения в коммутационных соотношениях алгебры возникает аномальный член (центральный заряд), в результате чего алгебра не замыкается и симметрия теряется.

Другие способы упорядочения недоступны в фоковскомпространстве, но могут иметь 
смысл в других пространствах состояний. Например, формальная замена ролей операторов рождения и уничтожения приводит к антифоковскому пространству, в котором определено антинормальное упорядочение квадрата массы. Вычисления показьвают, что центральные заряды алгебры Вирасоро в фоковском и антифоковском пространствах имеют противоположные знаки. (Этот факт приведен в [4] в качестве упражнения для читателя, мы решим это упражнение в разделе 1.) Этим свойством можно воспользоваться для сокращения аномалий в 4-мерной теории струны. Мы определим вакуумное состояние, являюшееся фоковским для осцилляторов из одной пары измерений и антифоковским для другой пары. В пространстве состояний, построенном на этом вакууме, вклады разных измерений в центральный заряд компенсируются. При ковариантном квантовании струны в таком пространстве система связей оказывается совместной.

Квантование струны технически проще проводить при разрешенных связях, например в калибровке светового конуса. В этом подходе аномалии нарушают врашательную инвариантность теории. Аномалии возникают в коммутационных соотношениях поперечной алгебры Вирасоро, встроенной в генераторы врашений. В разделе 2 мы выберем пространство состояний, в котором поперечная алгебра Вирасоро имеет нулевой центральный заряд, и построим оператор орбитального момента струны, подчиняющийся коммутационным соотношениям алгебры $\mathrm{SO}(3)$.

Антифоковское пространство состояний индефинитно. Следствия этого факта подробно описаны в разделе 3 и резюмированы в заключении.

\section{1. АЛГЕБРА ВИРАСОРО С НУЛЕВЫМ ЦЕНТРАЛЬНЫМ ЗАРЯДОМ В ТЕОРИИ СТРУНЫ}

Для построения алгебры Вирасоро вводится бесконечный набор осцилляторных переменных $a_{k}$

$$
\left[a_{k}, a_{n}\right]=k \delta_{k,-n}, \quad k, n \in \mathbf{Z}, \quad a_{k}^{+}=a_{-k} .
$$

Генераторы алгебры Вирасоро определяются следующим образом:

$$
L_{k}=\frac{1}{2} \sum_{n} a_{k-n} a_{n}, \quad k \neq 0, \quad L_{0}=\frac{1}{2} \sum_{n}: a_{-n} a_{n}: .
$$

При $k \neq 0$ операторы в $L_{k}$ коммутируют. Выполнены соотношения

$$
L_{k}^{+}=L_{-k}, \quad\left[L_{k}, a_{m}\right]=-m a_{k+m}
$$

Символ : $a_{-k} a_{k}$ : означает, что операторы уничтожения стоят справа от операторов рождения. В качестве оператора уничтожения можно выбрать любой из операторов $a_{k}, a_{-k}$. Этот выбор определяет вакуум:

1. Фоковский вакуум $a_{k}\left|0_{1}\right\rangle=0, k>0$.

Состояния рождаются из вакуума операторами $a_{-k}, k>0$; пространство состояний положительно определено;

$$
: a_{-k} a_{k}:=\left\{\begin{array}{ll}
a_{-k} a_{k}, & k>0 \\
a_{k} a_{-k}, & k<0
\end{array}=a_{-k} a_{k}+k \theta(-k)\right.
$$

2. Антифоковский вакуум $a_{-k}\left|0_{2}\right\rangle=0, k>0$. 
Состояния рождаются из вакуума операторами $a_{k}, k>0$; пространство индефинитно, например, $\left\langle 0_{2}\left|a_{1}^{+} a_{1}\right| 0_{2}\right\rangle=-1$;

$$
: a_{-k} a_{k}:=\left\{\begin{array}{ll}
a_{k} a_{-k}, & k>0 \\
a_{-k} a_{k}, & k<0
\end{array}=a_{-k} a_{k}+k \theta(k) .\right.
$$

Вычислим коммутатор

$$
\left[L_{k}, L_{n}\right]=\frac{1}{2} \sum_{l}\left[L_{k}, a_{n-l} a_{l}\right] .
$$

Здесь $n$ может равняться нулю, с-числовая добавка к $a_{n-l} a_{l}$ не влияет на коммутатор,

$$
\left[L_{k}, L_{n}\right]=\frac{1}{2} \sum_{l}(l-n) a_{n+k-l} a_{l}-l a_{n-l} a_{k+l} .
$$

1. При $k \neq-n$ сомножители в произведениях коммутируют. При больших $|l|$ в каждом члене суммы найдется оператор уничтожения (для любого из вакуумов 1,2$)$. Рассмотрим состояния с конечным числом заполненных мод (FMS), в которых все моды с номерами начиная с некоторого $N$ находятся в вакуумном состоянии. При действии на такие состояния сумма обрывается при больших $|l|$, т.е. сумма конечна. Во втором слагаемом выполним сдвиг индекса $l \rightarrow l-k$ :

$$
\left[L_{k}, L_{n}\right]=(k-n) L_{k+n} .
$$

2. $k=-n,\left[L_{k}, L_{-k}\right]=\frac{1}{2} \sum_{l}(l+k) a_{-l} a_{l}-l a_{-k-l} a_{k+l}$.

Каждое из двух слагаемых этого ряда по отдельности расходится, например,

$$
\sum_{l=-\infty}^{\infty}(l+k) a_{-l} a_{l}\left|0_{1}\right\rangle=-\underbrace{\sum_{l=-\infty}^{0}(l+k) l}_{\text {расходится }} \cdot\left|0_{1}\right\rangle .
$$

Приведем каждое слагаемое к нормальному виду $a_{-k} a_{k}=: a_{-k} a_{k}:-k \theta(\mp k)($ верхний знак для вакуума 1 , нижний - для вакуума 2):

$$
\left[L_{k}, L_{-k}\right]=\frac{1}{2} \sum_{l}(l+k): a_{-l} a_{l}:-l: a_{-k-l} a_{k+l}:-l(l+k)(\theta(\mp l)-\theta(\mp(l+k))) .
$$

При действии на FMS нормально упорядоченные суммы обрываются на больших $|l|$, суммы конечны. Выполняя сдвиг индекса $l \rightarrow l-k$ во втором слагаемом, получим

$$
\begin{gathered}
{\left[L_{k}, L_{-k}\right]=2 k L_{0}+c_{k},} \\
c_{k}=-\frac{1}{2} \sum_{l} l(l+k)(\theta(\mp l)-\theta(\mp(l+k)))=\mp \frac{1}{2} \sum_{l} l(l+k)(\theta(-l)-\theta(-(l+k))) .
\end{gathered}
$$

Таким образом, центральный заряд меняет знак при замене вакуума $1 \leftrightarrow 2$,

$$
\theta(-l)-\theta(-(l+k))=\left\{\begin{array}{l}
1, \text { при }-k<l<0, \\
0, \text { в противном случае }
\end{array} \quad(k>0),\right.
$$




$$
\begin{aligned}
c_{k}=\mp \frac{1}{2} \sum_{l=-k}^{0} l(l+k) & = \pm \frac{1}{2} \sum_{j=0}^{k} j(k-j)= \pm \frac{1}{12} k\left(k^{2}-1\right), \\
{\left[L_{k}, L_{-k}\right] } & =2 k L_{0} \pm \frac{1}{12} k\left(k^{2}-1\right) .
\end{aligned}
$$

Использовано тождество

$$
\sum_{j=0}^{k} j(k-j)=\frac{1}{6} k\left(k^{2}-1\right) .
$$

В $d$-мерном пространстве вводится $d$ наборов осцилляторов $a_{k}^{\mu} . L_{k}$ является суммой $d$ одномерных генераторов

$$
L_{k}=\frac{1}{2} \sum_{\mu} \sum_{n} a_{k-n}^{\mu} a_{n}^{\mu}
$$

Осцилляторные переменные из разных измерений коммутируют, поэтому центральный заряд аддитивен. Если для всех измерений выбран вакуум 1, то

$$
c_{k}=\frac{d}{12} k\left(k^{2}-1\right) .
$$

ЗАМЕЧАНИЕ. При изменении метрики (пространство Евклида ↔ пространство Минковского) получаем

$$
L_{k}=\frac{1}{2} \sum_{n} \vec{a}_{k-n} \vec{a}_{n} \pm a_{k-n}^{0} a_{n}^{0} .
$$

Для $a_{k}^{0}$ также меняется знак коммутатора. Поэтому для вклада измерения $\mu=0$ в алгебру Вирасоро имеем

$$
\left[ \pm L_{k}^{(0)}, \pm L_{n}^{(0)}\right]= \pm(k-n) L_{k+n}^{(0)}+\delta_{k,-n} c_{k}
$$

Первый член получается в результате вычисления одного коммутатора, поэтому он меняет знак. Центральный заряд возник при приведении произведений к нормальной форме, коммутатор вычислялся дважды, поэтому центральный заряд не меняет знака.

Таким образом, при изменении метрики алгебра Вирасоро сохраняет свои коммутационные соотношения и численное значение центрального заряда.

Выбор одного типа вакуума для всех измерений продиктован требованием явной лоренш-ковариантности теории, что не является безусловно необходимым. Если $d$ четно, и для половины измерений вакуум имеет тип 1 , а для другой половины - тип 2 , то центральные заряды компенсируются,

$$
d=4, \quad a_{k}^{1}|0\rangle=a_{k}^{2}|0\rangle=a_{-k}^{3}|0\rangle=a_{-k}^{0}|0\rangle=0, \quad k>0, \quad c_{k}=0 .
$$

В пространстве состояний, построенном на таком вакууме, алгебра Вирасоро не имеет аномалии. При ковариантном квантовании струны появляется возможность наложить на состояние все связи

$$
\left.L_{n} \mid \text { phys }\right\rangle=0, \quad n \in \mathbf{Z} .
$$

Теория не является лоренц-ковариантной явно, $L_{n}$ являются лоренц-скалярами, $\left[L_{n}, M^{\mu \nu}\right]=0$ независимо от выбора вакуума. Поэтому физическое подпространство 
(2) лоренц-инвариантно. Лоренц-неинвариантным является только вакуум (1), легко проверить, что он не сохраняется при преобразованиях Лоренца:

$$
\begin{aligned}
M^{\mu \nu} & =\frac{1}{2} P^{[\mu} X^{\nu]}+\frac{i}{2} \sum_{k>0} \frac{1}{k} a_{-k}^{[\mu} a_{k}^{\nu]}, \quad P^{\mu}=\sqrt{\pi} a_{0}^{\mu}, \\
M^{13}|0\rangle & =\left(\frac{1}{2} P^{[1} X^{3]}+\frac{i}{2} \sum_{k>0} \frac{1}{k} a_{-k}^{1} a_{k}^{3}\right)|0\rangle \neq 0 .
\end{aligned}
$$

С другой стороны, вакуум не лежит в физическом подпространстве, например:

$$
\begin{aligned}
L_{2}|0\rangle & =\frac{1}{2} \sum_{n} a_{2-n}^{\mu} a_{n}^{\mu}|0\rangle= \\
& =\left(-a_{2}^{0} \frac{P^{0}}{\sqrt{\pi}}+a_{2}^{3} \frac{P^{3}}{\sqrt{\pi}}-\frac{1}{2}\left(a_{1}^{0}\right)^{2}+\frac{1}{2}\left(a_{1}^{3}\right)^{2}\right)|0\rangle \neq 0 .
\end{aligned}
$$

Физическое вакуумное состояние следует искать в физическом подпространстве ${ }^{1)}$.

Таким образом, квантование струны в ковариантном подходе не встречает принципиальных трудностей. Для решения задачи следует найти базис физического подпространства (2). Мы не будем решать эту задачу, вместо этого проведем квантование струны в калибровке светового конуса.

\section{2. КВАНТОВАНИЕ СТРУНЫ В КАЛИБРОВКЕ СВЕТОВОГО КОНУСА}

Вводится набор поперечных осцилляторов $a_{k}^{1}, a_{k}^{2}$. Определим новые осцилляторные переменные

$$
a_{k}=\frac{a_{k}^{1}+i a_{k}^{2}}{\sqrt{2}}, \quad a_{k}^{+}=\frac{a_{-k}^{1}-i a_{-k}^{2}}{\sqrt{2}}, \quad k \in \mathbf{Z}, \quad\left[a_{k}, a_{n}^{+}\right]=k \delta_{k n}
$$

Из двух наборов осцилляторов с условием $a_{k}^{i+}=a_{-k}^{i}$ построен один набор без такого условия.

Мы будем использовать калибровку светового конуса, связанную с системой покоя струны [5]: направление оси калибровки задано вектором $P_{\mu} / \sqrt{P^{2}}-e_{\mu}$, где $P_{\mu}-$ полньй импульс струны, $e_{\mu}$ - единичный пространственно-подобный вектор, ортогональный $P_{\mu}$. При этом $a_{0}=0$. В такой калибровке значительно упрощается вычисление аномалии,

\footnotetext{
1) Может оказаться, что в физическом подпространстве нет состояния с наименьшей “энергией” $P^{2}$. Заметим, что в классической механике выражение $P^{2} / 2 \pi=\sum_{n>0} \vec{a}_{n}^{*} \vec{a}_{n}-a_{n}^{0 *} a_{n}^{0}$ на поверхности всех связей $L_{n}=0$ может быть отрицательным (достаточно рассмотреть пример $a_{ \pm 1}^{0}=2$, $a_{0}^{1}=2, a_{ \pm 2}^{1}=1, a_{ \pm 2}^{2}= \pm i$, остальные $\left.a_{n}^{\mu}=0\right)$. Для положительности $P^{2}$ необходимо наложить дополнительное условие $a^{0}(\sigma)=\sum a_{n}^{0} e^{i n \sigma}>0 \forall \sigma$ (мировая линия конца струны монотонна по времени Минковского). Но тогда это условие придется наложить и в квантовой теории, что представляется крайне проблематичным. Заметим еще, что в стандартной ковариантной теории струны спектр $P^{2}$ ограничен снизу по той причине, что операторы $a_{n}^{0+} a_{n}^{0}$ в этой теории принимают оmрицательные собственные значения.
} 
кроме того, в группе Лоренца оказывается достаточным рассмотреть подгруппу вращений в системе покоя - при устранении аномалии из этой подгруппы исключается аномалия из полной группы Лоренца.

Для поперечной алгебры Вирасоро

$$
L_{k}=\sum_{[n \neq 0, k]} a_{n-k}^{+} a_{n}, \quad k \neq 0, \quad L_{0}=\sum_{n}: a_{n}^{+} a_{n}:
$$

Сомножители в $L_{k}, k \neq 0$, коммутируют,

$$
L_{k}^{+}=L_{-k}, \quad\left[L_{k}, a_{m}\right]=-m a_{m+k}, \quad\left[L_{k}, a_{m}^{+}\right]=m a_{m-k}^{+}
$$

Вакуум 1 в новых переменных:

$$
\left\{\begin{array} { l } 
{ a _ { k } ^ { 1 } | 0 _ { 1 } \rangle = 0 } \\
{ a _ { k } ^ { 2 } | 0 _ { 1 } \rangle = 0 }
\end{array} ( k > 0 ) \Longleftrightarrow \left\{\begin{array}{l}
a_{k}\left|0_{1}\right\rangle=0, \quad k>0, \\
a_{k}^{+}\left|0_{1}\right\rangle=0, \quad k<0 .
\end{array}\right.\right.
$$

$\mathrm{B} a_{k}, a_{k}^{+}$-обозначениях удобно использовать

Вакуум 3: $a_{k}\left|0_{3}\right\rangle=0$ для всех $k \in \mathbf{Z} \backslash\{0\}$.

Вакуум 3 не совпадает ни с одним из рассмотренных вакуумов:

$$
\left(a_{k}^{1}+i a_{k}^{2}\right)\left|0_{3}\right\rangle=0, \quad k \in \mathbf{Z} \backslash\{0\} .
$$

Пространство состояний индефинитно (при $k<0$ оператор $a_{k}^{+}$рождает состояния с отрицательной нормой), $: a_{k}^{+} a_{k}:=a_{k}^{+} a_{k}$. Все дальнейшие вычисления проводятся в этом пространстве.

Поперечная алгебра Вирасоро не имеет аномалии:

$$
\left[L_{k}, L_{-k}\right]=\sum_{l}\left[L_{k}, a_{l+k}^{+} a_{l}\right]=\sum_{l}(l+k) a_{l}^{+} a_{l}-l a_{k+l}^{+} a_{k+l} .
$$

Произведения нормально упорядочены, суммы конечны на FMS. Поэтому

$$
\left[L_{k}, L_{-k}\right]=2 k L_{0}
$$

Генераторы группы врашений струны в системе покоя определены выражениями [5]

$$
S_{3}=\sum_{k \neq 0} \frac{1}{k} a_{k}^{+} a_{k}, \quad S^{-}=\sqrt{\frac{2 \pi}{P^{2}}} \sum_{k \neq 0} \frac{1}{k} a_{k} L_{-k}, \quad S^{+}=\sqrt{\frac{2 \pi}{P^{2}}} \sum_{k \neq 0} \frac{1}{k} a_{k}^{+} L_{k} .
$$

Врашения струны, меняющие ее положение относительно неподвижной оси калибровки (третьей оси), сопровождаются репараметризациями. Их выполняет встроенная в $S^{ \pm}$ поперечная алгебра Вирасоро.

Импульс $P_{\mu}$ удовлетворяет условию массовой поверхности $P^{2} / 2 \pi-L_{0}=0$, которая рассматривается как связь и является гамильтонианом системы. 
Сомножители в $S^{ \pm}$коммутируют: $\left[a_{k}, L_{-k}\right]=k a_{0}=0\left(\left[a_{k}, \sum_{n \neq 0,-k} a_{n+k}^{+} a_{n}\right]=0\right.$, поскольку в сумме не появляется $a_{k}^{+}$),

$$
\left[a_{k}, S_{3}\right]=a_{k}, \quad\left[a_{k}^{+}, S_{3}\right]=-a_{k}^{+} .
$$

Вообше если $A$ - произведение $n_{1}$ операторов $a$ и $n_{2}$ операторов $a^{+}$, то $\left[A, S_{3}\right]=\left(n_{1}-\right.$ $\left.n_{2}\right) A$ :

$$
\begin{gathered}
{\left[L_{k}, S_{3}\right]=0, \quad\left[S^{-}, S_{3}\right]=S^{-}, \quad\left[S^{+}, S_{3}\right]=-S^{+},} \\
S_{3} S^{ \pm}=S^{ \pm}\left(S_{3} \pm 1\right), \quad S_{3} S^{ \pm}\left|S_{3}=m\right\rangle=(m \pm 1) S^{ \pm}\left|S_{3}=m\right\rangle,
\end{gathered}
$$

$S^{+}$повьшшает $S_{3}, S^{-}$понижает $S_{3}$.

Аномалия возникает в $\left[S^{-}, S^{+}\right]$. В традиционном подходе используется вакуум 1 , аномалия имеет неустранимую часть, вызванную аномальными членами в поперечной алгебре Вирасоро. При использовании вакуума 3 эти члены отсутствуют. Подробное вычисление аномалии можно найти в [6]. Результат вычисления:

$$
\left[S^{-}, S^{+}\right]=-2 S_{3}+1 \text {. }
$$

Оставшийся аномальный член устраняется переопределением $S_{3}-\frac{1}{2} \rightarrow S_{3}$. При этом $S_{3}$ имеет полуцелый спектр. Построенная квантовая механика обладает также другими необычными свойствами, к описанию которых мы переходим.

\section{3. ПРЕДСТАВЛЕНИЕ ГРУППЫ ВРАШЕНИЙ}

3.1. Общая характеристика представлений группы вращений в индефинитных пространствах состояний. Коммутационные соотношения алгебры $\mathrm{SO}(3)$ :

$$
\left[S^{-}, S_{3}\right]=S^{-}, \quad\left[S^{+}, S_{3}\right]=-S^{+}, \quad\left[S^{-}, S^{+}\right]=-2 S_{3} .
$$

Оператор Казимира:

$$
\begin{aligned}
\vec{S}^{2} & =\frac{1}{2}\left(S^{+} S^{-}+S^{-} S^{+}\right)+S_{3}^{2}= \\
& =S^{+} S^{-}+S_{3}\left(S_{3}-1\right)=S^{-} S^{+}+S_{3}\left(S_{3}+1\right) .
\end{aligned}
$$

Собственные значения $\vec{S}^{2}$ обозначим $S(S+1)$. При $S(S+1) \geq-1 / 4$ величина $S$ вещественна, можно положить $S \geq-1 / 2$.

ЗАмЕчАниЕ. Стандартное представление группы врашений ( $S$ - целые или полуцелые $\geq 0, S_{3}=-S \ldots S$ с шагом 1 ) получается при использовании коммутационных соотношений и положительной определенности пространства состояний. Используется следуюшее рассуждение.

Подействуем операторами $S^{+}, S^{-}$на произвольное состояние с определенными $S$ и $S_{3}$. Получим последовательность состояний с тем же $S$, для которых $S_{3}$ меняется с шагом 1. Из (4) имеем

$$
\left\langle S, S_{3}\left|S^{+} S^{-}\right| S, S_{3}\right\rangle=\left(S(S+1)-S_{3}\left(S_{3}-1\right)\right)\left\langle S, S_{3} \mid S, S_{3}\right\rangle,
$$




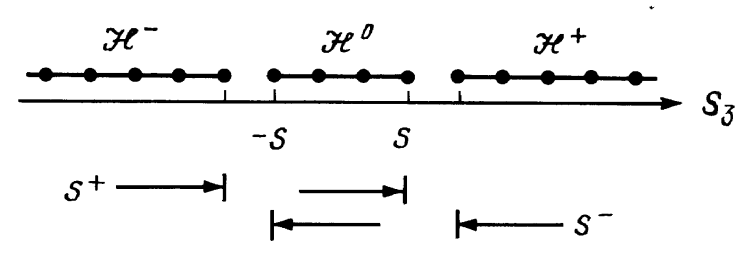

Рис. 1. Неприводимые врашательно-инвариантные пространства. $\mathcal{H}^{0}$ положительно определено, $\mathcal{H}^{-}$и $\mathcal{H}^{+}$индефинитны.

при больших $\left|S_{3}\right|$ коэффишиент в правой части становится отрицательным, поэтому либо $\left|S, S_{3}\right\rangle$, либо $S^{-}\left|S, S_{3}\right\rangle$ имеет отрицательную норму. Таких состояний нет в положительно определенном пространстве, поэтому последовательности $\left(S^{+}\right)^{k}\left|S, S_{3}\right\rangle$ и $\left(S^{-}\right)^{k}\left|S, S_{3}\right\rangle$ должны обрываться. Из (4) видно, что это возможно лишш при $S_{3}=$ $-S,-S+1, \ldots, S$.

В индефинитном пространстве приведенное рассуждение теряет силу, и группа вращений может иметь другие представления в этом пространстве. Охарактеризуем эти представления.

1. Простейшее представление имеет следующий вид.

Состояния: $|S m\rangle, 2 S \in \mathbf{Z}, S \geq-1 / 2, S_{3}=m=S+\mathbf{Z}$.

Скалярное произведение:

$$
\left\langle S^{\prime} m^{\prime} \mid S m\right\rangle=\delta_{S^{\prime} S} \delta_{m^{\prime} m} \cdot \begin{cases}1, & -S \leq m \leq S \\ (-1)^{m-S+1}, & m \geq S+1 \\ (-1)^{m+S+1}, & m \leq-S-1\end{cases}
$$

Операторы действуют на состояния следуюшим образом:

$$
S^{+}|S m\rangle=\alpha_{S m}^{+}|S, m+1\rangle, \quad S^{-}|S m\rangle=\alpha_{S m}^{-}|S, m-1\rangle,
$$

где

$$
\begin{aligned}
& \alpha_{S m}^{+}=\sqrt{|S(S+1)-m(m+1)|}, \\
& \alpha_{S m}^{-}=\alpha_{S, m-1}^{+} \text {при } \quad-S \leq m \leq S, \\
& \alpha_{S m}^{-}=-\alpha_{S, m-1}^{+} \quad \text { при } \quad m \geq S+1 \quad \text { или } \quad m \leq-S-1 .
\end{aligned}
$$

Легко убедиться, что выполнены все необходимые коммутационные соотношения и что $S^{+}$и $S^{-}$эрмитово сопряжены, а $S_{3}$ эрмитов. Выполнены соотношения

$$
S^{+}|S S\rangle=S^{+}|S,-S-1\rangle=0 \text { и } S^{-}|S,-S\rangle=S^{-}|S, S+1\rangle=0,
$$

поэтому все пространство состояний разбивается на прямую сумму пространств с $m \leq$ $-S-1,-S \leq m \leq S$ и $m \geq S+1$, инвариантных при действии всех генераторов врашений. Мы обозначим эти пространства $\mathcal{H}_{S}^{-}, \mathcal{H}_{S}^{0}$ и $\mathcal{H}_{S}^{+}$(рис. 1 ). (При $S=-1 / 2$ сектор $\mathcal{H}_{S}^{0}$ отсутствует.) В обшем случае нормы векторов $|S m\rangle$ не обязательно равны \pm 1 , как в (5). В каждом секторе скалярное произведение можно домножить на свой обший множитель без нарушения эрмитовых свойств операторов (6). 
Заметим, что спектр оператора $S_{3}$ в секторах $\mathcal{H}_{S}^{ \pm}$совпадает со спектром аналогичного оператора в представлениях группы Лоренца $\mathrm{SO}(2,1)$ дискретных серий $\mathcal{D}_{2 S+1}^{ \pm}{ }^{2}$. Это совпадение не случайно. Переход от алгебры $\mathrm{SO}(3)$ к алгебре $\mathrm{SO}(2,1)$ производится заменой операторов (поворотом Вика)

$$
S^{+} \rightarrow i S^{+}, \quad S^{-} \rightarrow i S^{-}, \quad S_{3} \rightarrow S_{3}
$$

и такой заменой скалярного произведения, чтобы новые операторы $S^{ \pm}$были эрмитово сопряжены:

$$
\left\langle S^{\prime} m^{\prime} \mid S m\right\rangle=\delta_{S^{\prime} S} \delta_{m^{\prime} m} \cdot \begin{cases}(-1)^{S+m}, & -S \leq m \leq S \quad(\text { cp. }(5)) \\ 1, & m \geq S+1, m \leq-S-1\end{cases}
$$

Линейное пространство представления при этом не меняется. При повороте Вика секторы $\mathcal{H}_{S}^{ \pm}$переходят в положительно определенные пространства $\mathcal{D}_{2 S+1}^{ \pm}$, сектору $\mathcal{H}_{S}^{0}$ отвечает индефинитное пространство, в котором реализуется конечномерное представление $\mathrm{SO}(2,1)$.

2. Имеются также другие представления группы врашений. Как видно из (4), последовательность $\left(S^{+}\right)^{k}|S m\rangle$ может обрываться только на состояниях $|S S\rangle$ и $|S,-S-1\rangle$, а $\left(S^{-}\right)^{k}|S m\rangle$ - только на $|S,-S\rangle$ и $|S, S+1\rangle$. Но последовательности не обязательно обрываются при прохождении этих состояний. Из (4) следует лишь

$$
S^{-} S^{+}|S S\rangle=0
$$

это означает, в частности, что $S^{+}|S S\rangle$ имеет нулевую норму. Все дальнейшие состояния в последовательности $\left(S^{+}\right)^{k}|S S\rangle$ также обладают нулевой нормой. На сумму таких представлений разлагаются произведения представлений $\mathcal{H}^{0} \otimes \mathcal{H}^{ \pm}$.

3. В общем случае $S_{3}-S$ и $2 S$ могут не быть целыми, возможно также $\vec{S}^{2}<-1 / 4$. Наконец, эрмитовы операторы $\vec{S}^{2}$ и $S_{3}$ могут принимать комплексные собственные значения на векторах с нулевой нормой [3]. С такими представлениями мы не встретимся.

Операторы конечных врашений. В секторах $\mathcal{H}_{S}^{ \pm}$генераторы врашений $S^{ \pm}$являются неограниченными операторами. Вследствие этого экспоненты от них определены не на всем пространстве состояний.

Рассмотрим оператор $e^{i \alpha S_{1}}$. Справедливо тождество [6]

$$
e^{i \alpha S_{1}}=e^{i \operatorname{tg} \alpha / 2 S^{+}}\left(\cos \frac{\alpha}{2}\right)^{-2 S_{3}} e^{i \operatorname{tg} \alpha / 2 S^{-}} .
$$

Подействуем оператором $e^{i \alpha S_{1}}$ на нижнее состояние сектора $\mathcal{H}_{S}^{+}$:

$$
e^{i \alpha S_{1}}|S, S+1\rangle=\left(\cos \frac{\alpha}{2}\right)^{-2(S+1)} \sum_{k=0}^{\infty} \frac{(i \operatorname{tg} \alpha / 2)^{k}}{k !}\left(\prod_{p=1}^{k} \alpha_{S, S+p}^{+}\right)|S, S+k+1\rangle .
$$

\footnotetext{
${ }^{2)}$ При полуцелых $S$ в пространствах $\mathcal{D}_{2 S+1}^{ \pm}$реализуются двузначные представления $\operatorname{SO}(2,1)$. Представления $\mathcal{D}_{0}^{ \pm}$называются ложнодискретными.
} 
Норма этого состояния равна

$$
(1+u)^{2(S+1)} \sum_{k=0}^{\infty} \frac{(2 S+k+1) !}{k !(2 S+1) !}(-u)^{k}
$$

где $u=\operatorname{tg}^{2} \alpha / 2$. Ряд в последнем выражении представляет разложение Тейлора функции $(1+u)^{-2(S+1)}$. Таким образом, внутри круга сходимости $|u|<1$ это выражение равно 1, а при $|u|>1$ ряд расходится. При $-\pi / 2<\alpha<\pi / 2$ состояние $e^{i \alpha S_{1}}|S, S+1\rangle$ обладает единичной нормой, при $\pi / 2<\alpha<\pi$ это состояние ненормируемо. Действие $e^{i \alpha S_{1}}$ при $\pi / 2<\alpha<\pi$ хорошо определено на состояниях вида $e^{-i \lambda S_{1}}|S, S+1\rangle$, $\alpha-\pi / 2<\lambda<\pi / 2$. На таких состояниях не определено действие $e^{-i \alpha S_{1}}, \pi / 2<\alpha<\pi$.

Итак, действие операторов конечных вращений определено не на всех состояниях каждьй оператор врашения имеет собственную область определения. В области определения эти операторы унитарны ${ }^{3)}$. Состояния вне области определения при вращениях переходят в ненормируемые векторы состояний (как элементы линейного пространства эти векторы вполне определены).

На то, что в секторе $\mathcal{H}_{S}^{+}$имеется препятствие к произвольным врашениям, указывает также следуюший факт: из формальных коммутационных соотношений вытекает, что вращение $e^{i \pi S_{1}}$ меняет знак $S_{3}$, с другой стороны, сектор $\mathcal{H}_{S}^{+}$содержит только состояния с $S_{3}>0$.

При действии оператора $e^{i \alpha S_{1}}$ на нижнее состояние сектора $\mathcal{H}_{S}^{0}$ получим состояние

$$
e^{i \alpha S_{1}}|S,-S\rangle=\left(\cos \frac{\alpha}{2}\right)^{2 S} \sum_{k=0}^{2 S} \frac{(i \operatorname{tg} \alpha / 2)^{k}}{k !}\left(\prod_{p=1}^{k} \alpha_{S,-S+p-1}^{+}\right)|S,-S+k\rangle
$$

обладающее нормой

$$
(1+u)^{-2 S} \sum_{k=0}^{2 S} \frac{(2 S) !}{k !(2 S-k) !} u^{k}=1
$$

Как и следовало ожидать, в секторе $\mathcal{H}_{S}^{0}$ определены все врашения.

Секторы $\mathcal{H}_{S}^{ \pm}$в точном смысле не являются пространствами представления группы врашений, они являются лишь пространствами, в которых реализована алгебра этой группы.

Мы рассмотрим также представления группы $\mathrm{SO}(2,1)$. Поворот Вика $S^{ \pm} \rightarrow i S^{ \pm}$ эквивалентен замене $\alpha \rightarrow i \alpha$ в формуле (7). Необходимо также учесть замену скалярного произведения. Проводя эти преобразования, получим формулу (8) с $u=-\operatorname{th}^{2} \alpha / 2$. При любом $\alpha$ значение $u$ находится внутри круга сходимости $|u|<1$, поэтому выражение (8) равно 1 , в пространствах $\mathcal{D}_{2 S+1}^{ \pm}$реализуется унитарное представление $\mathrm{SO}(2,1)$. Аналогичные преобразования для формулы (9) показывают, что конечномерное представление $\mathrm{SO}(2,1)$ псевдоунитарно.

\footnotetext{
3) В некоторых работах операторы, сохраняющие индефинитное скалярное произведение, называются псевдоунитарными.
} 
3.2. Представление алгебры $\mathrm{SO}(3)$ в теории струны. Вернемся к нашей задаче. Генераторы врашений определены выражениями

$$
\begin{gathered}
S_{3}=M-\frac{1}{2}, \quad M=\sum^{\prime} \frac{1}{k} a_{k}^{+} a_{k}, \\
S^{-}=\frac{1}{2} \sqrt{\frac{2 \pi}{P^{2}}} \sum^{\prime} \frac{k+n}{k n} a_{k} a_{n} a_{k+n}^{+}, \quad S^{+}=\frac{1}{2} \sqrt{\frac{2 \pi}{P^{2}}} \sum^{\prime} \frac{k+n}{k n} a_{k}^{+} a_{n}^{+} a_{k+n} .
\end{gathered}
$$

Необходимость симметризации, проведенной в $S^{ \pm}$, объясняется в [6]. Оператор квадрата массы струны: $L_{0}=\sum a_{k}^{+} a_{k}$.

Если $A$ - произведение операторов $a$ и $a^{+}$, то

$$
\left[A, L_{0}\right]=\left(\text { сумма индексов операторов } a-\text { сумма индексов операторов } a^{+}\right) \cdot A \text {, }
$$

поэтому

$$
\left[S_{3}, L_{0}\right]=0, \quad\left[S^{ \pm}, L_{0}\right]=0
$$

Состояния: $a_{k}^{+}|0\rangle=|k\rangle$, одно возбуждение в моде $k ; a_{k}|k\rangle=k|0\rangle ; a_{k}^{+} a_{l}^{+} \ldots a_{m}^{+}|0\rangle=$ $|k l \ldots m\rangle$, среди этих чисел могут быть совпадаюшие (несколько возбуждений в одной моде). Будем обозначать $\underbrace{k k \ldots k}_{n}$ через $n * k$,

$$
\begin{aligned}
a_{k}|n * k, \ldots\rangle & =k n|(n-1) * k, \ldots\rangle \\
\langle n * k, \ldots \mid n * k, \ldots\rangle & =k n\langle(n-1) * k, \ldots \mid(n-1) * k, \ldots\rangle= \\
& =k^{n} n !\langle\ldots \mid \ldots\rangle=\prod_{i} k_{i}^{n_{i}} n_{i} !
\end{aligned}
$$

Оператор $M$ :

$$
M\left|n_{1} * k_{1}, \ldots\right\rangle=\sum n_{i} \cdot\left|n_{1} * k_{1}, \ldots\right\rangle .
$$

$M$ подсчитывает число операторов $a^{+}$в состоянии:

$$
\begin{gathered}
a_{k}^{+} a_{l}^{+} \ldots a_{m}^{+}|0\rangle=|\underbrace{k l \ldots m}\rangle . \\
M=\text { количество этих чисел }
\end{gathered}
$$

Собственные значения $M \geq 0$. Собственные состояния $M$ :

$$
M=\begin{array}{ccccc}
|0\rangle, & |k\rangle, & |k l\rangle & \ldots \\
0, & 1, & 2, & \ldots
\end{array} .
$$

Оператор $L_{0}$ :

$$
L_{0}\left|n_{1} * k_{1}, \ldots\right\rangle=\sum k_{i} n_{i} \cdot\left|n_{1} * k_{1}, \ldots\right\rangle .
$$

$L_{0}$ подсчитывает сумму индексов $a^{+}$в состоянии:

$$
\begin{gathered}
a_{k}^{+} a_{l}^{+} \ldots a_{m}^{+}|0\rangle=|\underbrace{k l \ldots m}\rangle . \\
L_{0}=\text { сумма этих чисел }
\end{gathered}
$$




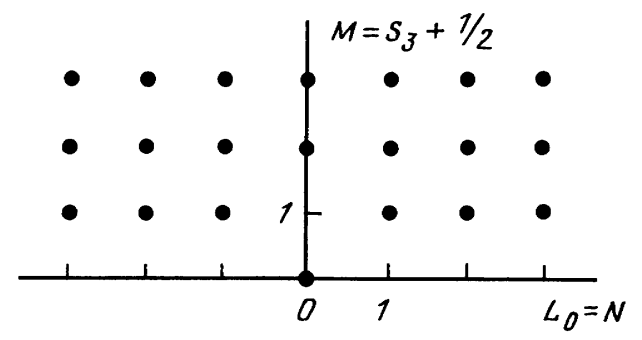

Рис. 2. Спектр $\left(L_{0}, M\right)$. Состояние $N=0, M=0$ невырожденно: $|0\rangle$. Состояния $N \neq 0, M=1$ невырожденны: $|N\rangle$. Состояния $N, M>1$ вырожденны с бесконечной кратностью (различные наборы $M$ чисел из $\mathbf{Z} \backslash\{0\}$, сумма которых равна $N): M=2:|k, N-k\rangle ; M=3:|k, l, N-k-l\rangle$ и т.д.

Собственные значения $L_{0} \in \mathbf{Z}$. Собственные состояния $L_{0}$ :

$$
\begin{array}{ccccc}
L_{0}=0: \quad|0\rangle, \quad|k,-k\rangle, \quad|k, l,-k-l\rangle, & \ldots
\end{array}
$$

Спектр $\left(L_{0}, M\right)$ приведен на рис. 2. Этот спектр имеет необычный вид, поскольку оператор $a_{k}^{+} a_{k}$ при $k<0$ принимает отрицательные собственные значения, например

$$
a_{-1}^{+} a_{-1} \cdot\left(a_{-1}^{+}\right)^{n}|0\rangle=-n \cdot\left(a_{-1}^{+}\right)^{n}|0\rangle
$$

так как $\left[a_{-1}, a_{-1}^{+}\right]=-1$.

Собственные состояния $\overrightarrow{\mathbf{S}}^{2}$. Поскольку операторы $S^{ \pm}$содержат множитель $\sqrt{2 \pi / P^{2}}$, они являются эрмитово сопряженными только при $P^{2}>0$. В дальнейшем мы будем рассматривать только такие состояния.

Рассмотрим 1-модовые состояния $|N\rangle, N>0$. Эти состояния аннигилируются понижающим оператором $S^{-}$, так как он содержит два оператора уничтожения. С другой стороны, на этих состояниях $M=1$, т.е. $S_{3}=1 / 2$. Из (4) заключаем, что $\vec{S}^{2}=-1 / 4$, т.е. $S=-1 / 2$. Повышающий оператор $S^{+}$содержит один оператор уничтожения, переводяший $|N\rangle$ в $|0\rangle$, и два оператора рождения, создаюших из $|0\rangle$ 2-модовое состояние с $S=-1 / 2, S_{3}=3 / 2$. Последовательными применениями $S^{+}$к $|N\rangle$ получаем базис состояний пространства $\mathcal{H}_{-1 / 2}^{+}$. Это построение продолжено в [6]. Результат изображен на рис. 3 . Все состояния содержатся в секторах $\mathcal{H}_{S}^{+}$.

Состояния с заданными $M=S_{3}+1 / 2$ и $N=P^{2} / 2 \pi$ имеют вид

$$
\Psi=\sum_{p_{1} \ldots p_{M}} c_{p_{1} \ldots p_{M}}\left|p_{1} \ldots p_{M}\right\rangle
$$

где коэффициент при состоянии симметричен по всем индексам и отличен от нуля только при $\sum_{i=1}^{M} p_{i}=N$.

Нижнее состояние в секторе $\mathcal{H}_{S}^{+}: \Psi=|S, S+1\rangle$ удовлетворяет условию

$$
\underset{q p_{3} \ldots p_{M}}{\operatorname{sym}} q \sum_{p} c_{q-p, p, p_{3} \ldots p_{M}}=0
$$




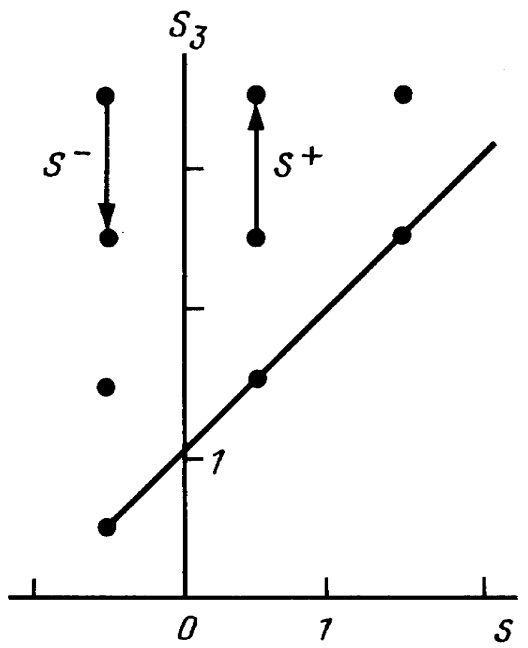

Рис. 3. Спектр $\left(S, S_{3}\right)$. Для каждого $L_{0}=N>0: S=-\frac{1}{2}, \frac{1}{2}, \frac{3}{2}, \ldots ; S_{3}=S+$ $1, S+2, \ldots$. Состояния с $S=-\frac{1}{2}$ невырожденны. Состояния с $S \geq \frac{1}{2}$ вырожденны с бесконечной кратностью.

Здесь sym означает симметризацию по перестановкам указанных индексов. Норма состояния равна

$$
\langle\Psi \mid \Psi\rangle=M ! \sum_{p_{1} \ldots p_{M}}\left|c_{p_{1} \ldots p_{M}}\right|^{2} p_{1} \ldots p_{M}
$$

Остальные состояния рождаются из $|S, S+1\rangle$ операторами $\left(S^{+}\right)^{k}$.

\section{ЗАКЛЮЧЕНИЕ}

В индефинитном пространстве состояний, использованном в этой работе, квантовая теория струны не имеет аномалий. При ковариантном квантовании струны система связей совместна, при квантовании в калибровке светового конуса алгебра вращений замкнута. Вместе с тем теория обладает следующими свойствами:

1. Представления алгебры $\mathrm{SO}(3)$ изоморфны представлениям $\mathcal{D}_{2 S+1}^{+}$алгебры $\mathrm{SO}(2,1)$. Для всех состояний вьполняется неравенство $S_{3}>S$.

2. Операторы конечных врашений определены не на всем пространстве состояний.

3. Нарушается классическое неравенство $P^{2} \geq 2 \pi S$ (условие Редже).

4. Теория не имеет вероятностной интерпретации $\left.{ }^{4}\right)$.

Кроме того, в пространстве состояний нет подпространств, в которых могла бы содержаться физически приемлемая теория. Построение теории с более реалистическими свойствами возможно при некоторых модификациях приведенной здесь конструкции.

1. В разделе 1 показано, что центральный заряд не зависит от метрики пространства Минковского. Отсюда следует, что в пространстве размерности $d=N+N$, содержащем

\footnotetext{
4) Проблемы вероятностной интерпретации квантовых теорий в индефиниитном пространстве иногда удается решить, см. [3].
} 
равное число пространственных и временных измерений

$$
\left[a_{k}^{\mu}, a_{n}^{\nu}\right]=g^{\mu \nu} k \delta_{k,-n}, \quad g^{\mu \nu}=\operatorname{diag}(\underbrace{+1 \cdots+1}_{N} \underbrace{-1 \ldots-1}_{N}),
$$

при выборе вакуума

$$
a_{k}^{i}|0\rangle=a_{-k}^{i+N}|0\rangle=0, \quad i=1, \ldots, N, \quad k>0
$$

центральный заряд равен нулю. При таком выборе вакуума пространство состояний положительно определено, поэтому в нем реализуются лишь стандартные представления группы Лоренца SO(N,N). Представляет интерес проведение компактификации “лишних" измерений в этой теории до $d=3+1$.

2. Сокращение аномалии в индефинитном фоковском пространстве раздела 1 не является специфическим свойством этого пространства, а имеет более глубокую природу. Покажем, каким образом можно реализовать алгебру Вирасоро с нулевым центральным зарядом в другом, более широком пространстве состояний.

Канонические переменные [7]:

$$
\left[Q_{\mu}(\sigma), Q_{\nu}\left(\sigma^{\prime}\right)\right]=2 i g_{\mu \nu} \vartheta\left(\sigma-\sigma^{\prime}\right)
$$

где $\vartheta(\sigma)=[\sigma / 2 \pi]+\frac{1}{2},[x]-$ целая часть $x, \quad \vartheta(\sigma)^{\prime}=\Delta(\sigma)-$ периодическая дельта-функция. Рассмотрим линейные комбинации

$$
Q_{ \pm}=\left(Q_{3} \pm Q_{0}\right) / \sqrt{2}, \quad Q=\left(Q_{1}+i Q_{2}\right) / \sqrt{2}, \quad Q^{*}=\left(Q_{1}-i Q_{2}\right) / \sqrt{2},
$$

для них имеем

$$
\left[Q_{-}(\sigma), Q_{+}\left(\sigma^{\prime}\right)\right]=-2 i \vartheta\left(\sigma-\sigma^{\prime}\right), \quad\left[Q^{*}(\sigma), Q\left(\sigma^{\prime}\right)\right]=-2 i \vartheta\left(\sigma-\sigma^{\prime}\right)
$$

остальные коммутаторы равны нулю.

$\left(Q_{+}, Q\right)$ образуют полный набор коммутируюших переменных квантовой теории. В качестве пространства состояний выберем линейное пространство функционалов $\Psi\left(Q_{+}, Q\right)$. Переменные $Q_{+}$и $Q$ реализуем мультипликативными операторами, $Q_{-}^{\prime}$ и $Q^{\prime *}$ - операторами дифференцирования ${ }^{5)}$ :

$$
\begin{aligned}
Q_{-}^{\prime}(\sigma) & =-2 i \frac{\delta}{\delta Q_{+}(\sigma)}, & Q^{\prime *}(\sigma) & =-2 i \frac{\delta}{\delta Q(\sigma)}, \\
{\left[Q_{-}^{\prime}(\sigma), Q_{+}\left(\sigma^{\prime}\right)\right] } & =-2 i \Delta\left(\sigma-\sigma^{\prime}\right), & {\left[Q^{\prime} *(\sigma), Q\left(\sigma^{\prime}\right)\right] } & =-2 i \Delta\left(\sigma-\sigma^{\prime}\right) .
\end{aligned}
$$

\footnotetext{
${ }^{5)}$ Используя вместо операторов $Q_{\mu}(\sigma)$ коэффициенты их разложений Фурье и ограничив пространство функционалов $\Psi\left(Q_{+}, Q\right)$ на полиномиальные функции от коэффициентов Фурье, получим фоковское пространство состояний раздела 1 . Заметим, что генератор врашений $Q \rightarrow Q e^{i \varphi}$ принимает в этом пространстве только неотрицательные собственные значения. Пространство функционалов $\Psi\left(Q_{+}, Q\right)$ шире, чем фоковское, в частности оно содержит состояния отрицательной степени однородности по $Q$.
} 
Нетрудно также построить оператор средней координаты и определить $Q_{-}$и $Q^{*}$ так, чтобы выполнялись коммутационные соотношения (11). Здесь нам потребуются только производные $Q_{-}^{\prime}$ и $Q^{\prime *} *$.

В генераторе репараметризаций выберем следующее упорядочение:

$$
Q_{\mu}^{\prime 2}=-2\left(Q_{+}^{\prime} Q_{-}^{\prime}+Q^{\prime} Q^{\prime *}\right) \sim Q_{+}^{\prime} \frac{\delta}{\delta Q_{+}(\sigma)}+Q^{\prime} \frac{\delta}{\delta Q(\sigma)}=L(\sigma)
$$

Легко убедиться, что этот оператор действительно генерирует репараметризации $\left(Q_{+}(\sigma), Q(\sigma)\right)$ в аргументе $\Psi$ :

$$
\left.\frac{\delta}{\delta \varepsilon(\sigma)} \Psi\left(Q_{+}(\sigma+\varepsilon(\sigma)), Q(\sigma+\varepsilon(\sigma))\right)\right|_{\varepsilon=0}=\frac{\delta \Psi}{\delta Q_{+}(\sigma)} Q_{+}^{\prime}(\sigma)+\frac{\delta \Psi}{\delta Q(\sigma)} Q^{\prime}(\sigma)=L(\sigma) \Psi .
$$

Поэтому алгебра операторов $L(\sigma)$ замкнута. Это также можно проверить непосредственным вычислением.

Решениями связей $L(\sigma) \Psi=0$ являются параметрически-инвариантные функционалы $\Psi\left(Q_{+}(\sigma), Q(\sigma)\right)$ (параметрические инварианты кривой $\left(Q_{+}(\sigma), Q(\sigma)\right) \subset \mathbf{R} \times \mathbf{C}$, голоморфные по $Q)$. Примерами таких инвариантов являются

$$
\phi=\int_{0}^{2 \pi} d \sigma\left(F\left(Q_{+}, Q\right) Q_{+}^{\prime}+G\left(Q_{+}, Q\right) Q^{\prime}\right)
$$

а также произвольные функции от них.

Таким образом, алгебру Вирасоро с нулевым центральным зарядом можно представить в линейном пространстве состояний, не наделенном скалярным произведением. Следующим шагом должно стать введение такого скалярного произведения, по отношению к которому операторы $Q$ и $Q^{*}$ эрмитово сопряжены, а $Q+$ и $Q$ - эрмитовы. Это требование, возможно, является избыточным, поскольку операторы $Q_{\mu}$ не коммутируют со связями, не сохраняют физическое пространство и поэтому не являются наблюдаемыми. В квантовых теориях с некомпактной калибровочной группой в расширенном и физическом пространствах приходится вводить два различных скалярных произведения, поскольку скалярное произведение расширенного пространства расходится на физических векторах (см. [8]). Безусловно необходимым является вьполнение эрмитовых свойств по отношению к физическому скалярному произведению для операторов, действуюших в физическом пространстве. Желательно также, чтобы физическое скалярное произведение было положительно определено. Свойства скалярного произведения в расширенном пространстве не влияют на физические следствия теории.

Автор выражает благодарность за полезные обсуждения Е. Б. Бердникову, Г. П. Пронько, А. В. Разумову и Л. Д. Соловьеву. Работа выполнена при финансовой поддержке Международной соросовской программы, грант № а734-фю.

\section{Список литературы}

[1] Dirac P. A. M. // Comm. Dublin Inst. Adv. Studies. 1943. P. 1.

[2] Pauli W. // Rev.Mod.Phys. 1943. V. 15. P. 175.

[3] Надь K. Пространства состояний с индефинитной метрикой в квантовой теории поля. М.: Мир, 1969

[4] Бринк Л., Энно М. Принципы теории струн. М.: Мир, 1991. С. 156. 
[5] Никитин И. Н. // ЯФ. 1993. Т. 56. №9. С. 230.

[6] Никитин И. Н. Квантовая теория струны в индефинитном пространстве состояний: Препринт ИФВЭ 95-113. Протвино: ИФВЭ, 1995.

[7] Pron’ko G. P. // Rev. Math. Phys. 1990. V. 2. № 3. P. 355.

[8] Никитин И. Н., Пронько Г. П. Электромагнитное взаимодействие в теории прямолинейной струны: Препринт ИФВЭ 94-64. Протвино: ИФВЭ, 1994.

Институт физики

высоких энергий

Поступила в редакцию 27.IX.1995 г

\section{N. Nikitin}

\section{QUANTUM STRING THEORY IN INDEFINITE SPACE OF STATES}

The quantum string theory in indefinite (pseudo-Hilbert) Fock representation is considered. It is shown that all anomalies in 4-dimensional theory of open bosonic string are cancelled at particular choice of vacuum state. In this the algebra of reparametrizations (in covariant approach) and the algebra of rotations (in light cone gauge) become closed. The indefiniteness of the state space introduces some non-physical features into the theory. Modifications of the given method are discussed, which are necessary to construct more realistic theory. 